\title{
Comparação de métodos de regressão para avaliar a estabilidade fenotípica em cana-de-açúcar ${ }^{(1)}$
}

\begin{abstract}
Leonardo Novaes Rosse ${ }^{(2)}$, Roland Vencovsky(3) e Daniel Furtado Ferreira(4)
Resumo - O objetivo deste trabalho foi comparar dois métodos de regressão empregados para avaliar a estabilidade fenotípica em plantas de cana-de-açúcar, o linear, de Cruz et al., e o não-linear, de Toler \& Burrows. Utilizaram-se as informações do ensaio de caracterização de 1994 do programa de melhoramento genético da Copersucar, compreendendo 13 locais e 20 genótipos de cana-de-açúcar no Estado de São Paulo. Os resultados permitiram concluir que ambos os modelos se ajustaram satisfatoriamente aos dados. As estimativas da qualidade ambiental pelos dois modelos mostraram diferenças entre seus valores, porém foram concordantes para inferir sobre os melhores e piores ambientes. Ao classificar os genótipos com base na produtividade e no padrão de resposta, no entanto, apenas dez foram coincidentes nos dois métodos. As vantagens estatísticas do método não-linear torna-o recomendável para pesquisa sobre estabilidade.
\end{abstract}

Termos para indexação: Saccharum, interação genótipo-ambiente, modelos, análise de regressão.

\section{Comparison of regression methods for evaluation of phenotypic stability in sugarcane}

Abstract - The purpose of this work was to compare the regression methods used for evaluating phenotypic stability in plants. The methods of Cruz et al. based on a linear model and of Toler \& Burrows based on a non-linear model were considered. Data of the sugarcane 1994 characterization trial coordinated by the genetic improvement program of Copersucar were used. Experiments were set up in 13 locations of the State of São Paulo, in Brazil for evaluation of 20 sugarcane genotypes. The fitting of the models to the data was obtained for both methods. Measures of environmental quality were numerically different but agreed in general terms for the ranking of locations. However, when genotypes were classified on the bases of yield and pattern of response only ten coincided for both methods. The statistical advantages of the non-linear model makes it recommendable for studies on stability.

Index terms: Saccharum, genotype environment interaction, models, regression analysis.

\section{Introdução}

A cana-de-açúcar (Saccharum spp.) é um produto de suma importância econômica e social no Brasil, em virtude dos seus derivados, principalmente o álcool e o açúcar. O Estado de São Paulo destaca-se por contribuir com cerca de $60 \%$ da produção nacional, o que

(1) Aceito para publicação em 23 de abril de 2001

Extraído da Tese de Doutorado apresentada pelo primeiro autor à Escola Superior de Agricultura Luiz de Queiroz (Esalq), Piracicaba, SP.

(2) Universidade Federal do Paraná (UFPR), Dep. de Estatística, Caixa Postal 19081, CEP 81530-990 Curitiba, PR. E-mail: lnrosse@est.ufpr.br

(3) Esalq, Dep. de Genética, Caixa Postal 83, CEP 23400- 970 Piracicaba, SP. E-mail: vencovsk@zaz.com.br

(4) Universidade Federal de Lavras, Dep. de Ciências Exatas, Caixa Postal 37, CEP 37200-000 Lavras, MG. E-mail: danielff@ufla.br equivale a aproximadamente 300 milhões de toneladas anuais (Raizer, 1998).

O sucesso dessa atividade econômica está atrelado principalmente aos programas de melhoramento genético, nos quais um dos objetivos é desenvolver variedades adaptadas às diversas regiões canavieiras, visando contornar problemas relacionados a ataques de pragas e resistência às doenças, além de melhorar as características industriais das variedades.

O Estado de São Paulo, por sua extensão territorial, mostra elevada diversidade edafoclimática entre as principais regiões produtoras, fazendo com que a classificação das variedades de um ambiente para outro não seja a mesma. Esse fenômeno em que a classificação dos materiais é alterada, denomina-se interação de genótipos por ambientes e suas impli- 
cações nos programas de melhoramento já são bastante conhecidas e discutidas (Ramalho et al., 1993; Cruz \& Regazzi, 1994; Duarte \& Vencovsky, 1999).

Para atenuar os problemas gerados por essa interação, é conveniente que os melhoristas conheçam a sua magnitude, o que é possível mediante técnicas adequadas. Entre elas, pode-se citar a recomendação de materiais de maior estabilidade fenotípica por meio de modelos de regressão linear uni e bissegmentados (Eberhart \& Russell, 1966; Verma et al., 1978; Silva \& Barreto, 1985; Cruz et al., 1989; Storck \& Vencovsky, 1994). Desses métodos, o de Cruz et al. (1989) tem sido um dos mais empregados, por apresentar ausência de correlação entre as estimativas dos parâmetros que avaliam a adaptabilidade dos genótipos. Apesar do uso generalizado desses modelos, eles apresentam algumas restrições de cunho estatístico. A mais citada relaciona-se com a variável independente, representada pelo índice ambiental. Essa variável que mensura a qualidade do ambiente é estimada com os próprios dados dos genótipos. Esse processo de estimação, como tal, viola, os princípios básicos da análise de regressão.

Uma das alternativas para contornar esse inconveniente seria o emprego de modelo de regressão não-linear nos parâmetros, como o apresentado por Toler \& Burrows (1998). Segundo esses autores, o modelo é, estatisticamente, um aprimoramento dos modelos lineares de estabilidade, por contornar os problemas relacionados com a estimativa do índice ambiental, fazendo com que os testes das hipóteses para os seus parâmetros sejam realizados sem violar os princípios básicos necessários para a sua validade.

A comparação dos parâmetros de estabilidade e adequação dos modelos visando contornar problemas com seus estimadores é uma das preocupações que os melhoristas enfrentam na escolha do método a empregar. Estudos enfocando esses aspectos são encontrados em Duarte \& Zimmermann (1992), Silva (1995, 1998) e Storck (1998).

O objetivo deste trabalho foi comparar dois métodos de regressão para avaliar a estabilidade fenotípica em plantas de cana-de-açúcar: o linear de Cruz et al., e o não-linear de Toler \& Burrows.

\section{Material e Métodos}

Avaliaram-se dados de produtividade de açúcar (TPH) estimados para cinco cortes em 13 regiões produtoras re- presentativas do Estado de São Paulo no ano de 1994 (Tabela 1). Esses ensaios corresponderam à última fase de experimentação do programa de melhoramento da Copersucar (Raizer, 1998), em que se empregou o delineamento em blocos completos ao acaso, com quatro repetições. As parcelas úteis foram constituídas de cinco sulcos de largura por $12 \mathrm{~m}$ de comprimento, com espaçamento entre linhas de 1,10 a 1,50 m, e a área da parcela variando de 66 a $90 \mathrm{~m}^{2}$.

Realizada a análise conjunta da variância e detectada significância para a fonte de variação interação genótipos $\mathrm{x}$ locais, procedeu-se à análise de estabilidade fenotípica. Utilizaram-se os modelos de regressão linear nos parâmetros de Cruz et al. (1989) e o não-linear de Toler \& Burrows (1998), considerando p genótipos em q ambientes.

O modelo de Cruz et al. (1989), que se baseia na regressão linear múltipla, é expresso da seguinte forma:

$Y_{i j}=b_{0 i}+b_{1 i} I_{j}+b_{2 i} T\left(I_{j}\right)+\delta_{i j}+\bar{\varepsilon}_{i j}$ em que:

$\mathrm{Y}_{\mathrm{ij}}$ : é a produtividade média do genótipo i no ambiente $\mathrm{j}$; $\mathrm{b}_{0 \mathrm{i}}$ : é a produtividade média geral do genótipo i ao longo de todos os ambientes;

$\mathrm{b}_{1 \mathrm{i}}$ : é o coeficiente de regressão linear que dá a resposta do genótipo i à variação nos ambientes desfavoráveis;

$\mathrm{I}_{\mathrm{j}}$ : índice ambiental, dado por $\mathrm{I}_{\mathrm{j}}=\overline{\mathrm{Y}}_{\mathrm{j}}-\overline{\mathrm{Y}}_{\text {; }}$;

$\mathrm{b}_{2 \mathrm{i}}$ : é o coeficiente de regressão linear que informa sobre

Tabela 1. Relação dos genótipos e dos locais de avaliação que compuseram o ensaio de caracterização de 1994 do programa de melhoramento de cana-de-açúcar da Copersucar, no Estado de São Paulo.

\begin{tabular}{|c|c|c|}
\hline Usina & Município & Genótipos \\
\hline Barra Grande & Lençóis Paulista & 1: RB72454 $4^{(1)}$ \\
\hline Catanduva & Ariranha & 2: SP70-1143 ${ }^{(1)}$ \\
\hline Cresciumal & Leme & 3: SP78-4792 \\
\hline Diamante & Jaú & 4: SP79-1011 ${ }^{(1)}$ \\
\hline Santa Adélia & Jaboticabal & 5: SP79-2312 $12^{(1)}$ \\
\hline Santa Luiza & Araraquara & 6: SP80-0085 \\
\hline Santa Maria & Cerquilho & 7: SP80-0144 \\
\hline São João & Araras & 8: SP80-0180 \\
\hline São Luiz AA & Pirassununga & 9: SP80-3280 \\
\hline São Luiz SA & Ourinhos & 10: SP80-3390 \\
\hline São Manoel & São Manoel & 11: SP80-3862 \\
\hline São Martinho & Pradópolis & 12: SP80-4439 \\
\hline \multirow[t]{8}{*}{ Zanin } & Araraquara & 13: SP80-4445 \\
\hline & & 14: SP80-4966 \\
\hline & & 15: SP81-1776 \\
\hline & & 16: SP82-1724 \\
\hline & & 17: SP82-3530 \\
\hline & & 18: SP82-4055 \\
\hline & & 19: SP82-6108 \\
\hline & & 20: SP83-1483 \\
\hline
\end{tabular}


o diferencial de resposta do genótipo i à variação nos ambientes favoráveis;

$\mathrm{T}\left(\mathrm{I}_{\mathrm{j}}\right)=0$, se $\mathrm{I}_{\mathrm{j}} \leq 0$;

$\mathrm{T}\left(\mathrm{I}_{\mathrm{j}}\right)=\mathrm{I}_{\mathrm{j}}-\overline{\mathrm{I}}_{+}$, se $\mathrm{I}_{\mathrm{j}}>0$, sendo $\overline{\mathrm{I}}_{+}$a média dos índices $\mathrm{I}_{\mathrm{j}}$ positivos

$\delta_{\mathrm{ij}}$ : é o desvio da regressão do genótipo i no ambiente $\mathrm{j}$;

$\bar{\varepsilon}_{\mathrm{ij}}$ : é o erro experimental médio, suposto normal e independentemente distribuído NID $\left(0, \sigma^{2}\right)$.

O coeficiente de determinação $\left(\mathrm{R}^{2}\right)$, que informa sobre o ajustamento do modelo aos dados, foi estimado conforme Cruz \& Regazzi (1994).

As significâncias estatísticas relativas aos parâmetros foram obtidas pelo teste t por meio das seguintes hipóteses:

$\mathrm{H}_{01}: \mathrm{b}_{1 \mathrm{i}}=1$

$\mathrm{H}_{02}: \mathrm{b}_{2 \mathrm{i}}=0$

$\mathrm{H}_{03}: \mathrm{b}_{1 \mathrm{i}}+\mathrm{b}_{2 \mathrm{i}}=1$

O modelo de regressão não-linear de Toler \& Burrows (1998) apresenta a seguinte notação:

$Y_{i j}=\alpha_{i}+\left[Z_{j} \beta_{1 i}+\left(1-Z_{j}\right) \beta_{2 i}\right] \mu_{j}+\varepsilon_{i j}$

em que:

$Y_{\mathrm{ij}}$ : é a resposta média do genótipo i no ambiente j;

$\alpha_{i}$ : é o parâmetro que reflete o valor da resposta do genótipo i no intercepto $\operatorname{com} \mu=0$, podendo assumir valor igual ou diferente da média geral do genótipo i conforme os testes das hipóteses;

$\beta_{1 \mathrm{i}}$ e $\beta_{2 \mathrm{i}}$ : são os parâmetros que refletem a sensibilidade de resposta do genótipo i nos ambientes de baixa e alta produtividade média, respectivamente;

$\mu_{\mathrm{j}}$ : é o parâmetro que reflete o efeito do ambiente $\mathrm{j}$;

$\varepsilon_{\mathrm{ij}}$ : é o erro experimental médio, suposto normal e independentemente distribuído $\operatorname{NID}\left(0, \sigma^{2}\right)$.

$Z_{\mathrm{j}}=1$, se $\mu_{\mathrm{j}} \leq 0 \mathrm{e}$,

$Z_{\mathrm{j}}^{\mathrm{j}}=0$, se $\mu_{\mathrm{j}}^{\mathrm{j}}>0$.

O ajustamento do modelo aos dados foi avaliado por meio do coeficiente $\mathrm{R}^{2}$ e os testes das hipóteses dos parâmetros do modelo feitos pelo teste t (Rosse, 1999).

Com base nos testes das hipóteses dos parâmetros que informam sobre o padrão de resposta dos materiais, foi possível enquadrá-los em cinco grupos. Os critérios para esse enquadramento segundo Toler \& Burrows (1998) são apresentados por Rosse \& Vencovsky (2000). No caso do modelo de Cruz et al. (1989), os critérios adotados foram os seguintes. Quando se aceitou a hipótese de que $b_{1 i}=1 \mathrm{e}$ $\mathrm{b}_{1 \mathrm{i}}+\mathrm{b}_{2 \mathrm{i}}=1$, o genótipo i enquadrar-se-á no grupo $\mathrm{C}$ (não-significância em relação $\mathrm{a} b=1$ ). Se os testes levam à aceitação das hipóteses alternativas $b_{1 i}>1$ e $b_{1 i}+b_{2 i}>1$ (significância em relação a b=1), o material i classificar-se-á no grupo B. Quando $b_{1 i}<1$ e $b_{1 i}+b_{2 i}<1$, a classificação é no grupo $D$. Se for aceito que $b_{1 i}=1$ e $b_{1 i}+b_{2 i}>1$, o material alocar-se-á no grupo A e, finalmente, se $b_{1 \mathrm{i}}=1$ e $b_{1 \mathrm{i}}+b_{2 \mathrm{i}}<1$, no grupo E.

O significado prático de cada grupo, segundo Toler \& Burrows (1998) é:

A: resposta convexa e duplamente desejável;

B: resposta linear simples e desejável em ambientes de alta qualidade;

C: resposta linear simples não desviando da resposta média dos ambientes;

D: resposta linear simples e desejável em ambientes de baixa qualidade;

E: resposta côncava e duplamente indesejável.

\section{Resultados e Discussão}

A análise de variância conjunta indicou diferenças altamente significativas em relação às fontes de variação de genótipos, locais e da interação entre eles. Isso significa que os genótipos testados diferiram em produtividade de açúcar, bem como os locais, por causa dos fatores edafoclimáticos das localidades. A interação de genótipos com locais indica que os níveis de produtividade de açúcar dos genótipos nos vários locais foram diferenciados. Assumindo que essa interação reflete também a existência de genótipos de adaptação específica e outros de adaptação geral, um estudo mais detalhado desse fenômeno a partir da análise de estabilidade e adaptabilidade seria justificado.

A significância dessas fontes de variação torna-se evidente quando se observa a precisão experimental dada pelo coeficiente de variação $(6,2 \%)$ que indica que os experimentos foram bem conduzidos.

Em relação ao efeito de comparação entre os modelos de regressão, os testes das hipóteses para os padrões de resposta nos ambientes favoráveis e desfavoráveis devem ser equivalentes. Assim, para o modelo linear bissegmentado, quando se consideram os genótipos nos ambientes desfavoráveis a hipótese a ser testada é $\mathrm{H}\left(\mathrm{b}_{1 \mathrm{i}}=1\right)$, e nos favoráveis $\mathrm{H}\left(\mathrm{b}_{1 \mathrm{i}}+\mathrm{b}_{2 \mathrm{i}}=1\right)$. A hipótese $\mathrm{H}\left(\mathrm{b}_{2 \mathrm{i}}=0\right)$ não rejeitada indica que apenas um segmento de reta é suficiente para expressar o comportamento de um dado genótipo. Caso contrário, testa-se a hipótese 
$\mathrm{H}\left(\mathrm{b}_{1 \mathrm{i}}+\mathrm{b}_{2 \mathrm{i}}=1\right)$. No caso do modelo de Toler \& Burrows (1998), a não-rejeição da hipótese $\mathrm{H}\left(\beta_{2 \mathrm{i}}-\beta_{1 \mathrm{i}}\right)=0$ fornece a mesma interpretação do modelo linear mencionado anteriormente, ou seja, de que não há diferença entre os ambientes favoráveis e desfavoráveis, e uma única linha reta explica satisfatoriamente o comportamento dos genótipos. Embora os testes se equivalham, o processo de estimação dos parâmetros é diferente. No linear, a estimação é feita através de quadrados mínimos, e no não-linear, pelo processo iterativo de quadrados mínimos (Gallant, 1987).

No de Cruz et al. (1989), o coeficiente $\mathrm{b}_{0 \mathrm{i}}$ é igual à média geral do genótipo $\mathrm{i}\left(\overline{\mathrm{Y}}_{\mathrm{i}}\right)$ (Tabela 2$)$. No modelo de Toler \& Burrows (1998), o estimador $\hat{\alpha}_{i}$ pode ser igual ou diferente de $\bar{Y}_{i .}$ para mais ou para menos, conforme os testes das hipóteses que irão determinar se o comportamento dos genótipos pode ser predito por um ou dois segmentos de reta.
Quando o melhor ajuste for por um único segmento de reta, $\hat{\alpha}_{i}=\bar{Y}_{i .}$. Caso contrário, $\hat{\alpha}_{i} \neq \bar{Y}_{i}$, e $\hat{\alpha}_{i}$ será maior que $\bar{Y}_{\text {i. }}$ quando $\hat{\beta}_{1 \mathrm{i}}>\hat{\beta}_{2 \mathrm{i}}$ ou $\hat{\alpha}_{\mathrm{i}}$ será menor que $\bar{Y}_{\mathrm{i} \text {. }}$, quando $\hat{\beta}_{1 \mathrm{i}}<\hat{\beta}_{2 \mathrm{i}}$. Essas duas últimas situações ocorreram em 50\% dos genótipos. Rosse (1999) mencionou um meio de minimizar tais diferenças, fazendo com que $\hat{\alpha}_{i}$ se aproxime de $\bar{Y}_{i}$. Ele propõe que a avaliação dos genótipos seja realizada preferencialmente em maior número possível de locais.

Com relação aos parâmetros que expressam os padrões de resposta dos genótipos, nos dois modelos, as respectivas estimativas foram de magnitudes diferentes (Tabela 2). Por outro lado, não há uma nítida diferença entre os valores estimados dos coeficientes de determinação $\left(\mathrm{R}^{2}\right)$. Estes apresentaram grau de correlação de 0,86 (Tabela 3 ). Em termos gerais, essa alta correlação entre os $\mathrm{R}^{2}$ dos genótipos dos dois métodos forneceram graus satisfatórios de ajustamento aos dados e não muito discrepantes.

Tabela 2. Médias observadas $\left(\overline{\mathrm{Y}}_{\mathrm{i}}\right.$ ) e estimativas dos parâmetros de estabilidade obtidas segundo o modelo de Toler \& Burrows (1998) e Cruz et al. (1989), para a produtividade de açúcar na avaliação de genótipos de cana-de-açúcar do ensaio de caracterização de 1994 da Copersucar, no Estado de São Paulo.

\begin{tabular}{|c|c|c|c|c|c|c|c|}
\hline \multirow[t]{2}{*}{ Genótipos } & \multirow[t]{2}{*}{$\bar{Y}_{\mathrm{i}}$} & \multicolumn{4}{|c|}{ Toler \& Burrows } & \multicolumn{2}{|c|}{ Cruz et al. } \\
\hline & & $\hat{\alpha}_{i}$ & $\hat{\beta}_{1 \mathrm{i}}$ & $\hat{\beta}_{2 \mathrm{i}}$ & $\hat{\beta}$ comum & $\hat{b}_{1}$ & $\hat{\mathrm{b}}_{1}+\hat{\mathrm{b}}_{2}$ \\
\hline 9 & 14,7 & $\begin{array}{l}14,7 \\
\end{array}$ & - & - & $1,19^{* * *}$ & $1,14 *$ & $2,07 * *$ \\
\hline 13 & 14,4 & 14,7 & $1,38^{* *}$ & $0,91^{\mathrm{ns}}$ & - & $1,14^{*}$ & $0,60^{\text {ns }}$ \\
\hline 10 & 14,3 & 14,8 & $1,53 * *$ & $0,80^{\mathrm{ns}}$ & - & $1,11^{\mathrm{ns}}$ & $1,21^{\mathrm{ns}}$ \\
\hline 1 & 14,0 & 15,2 & $1,96^{* *}$ & $0,24 * *$ & - & $1,08^{\mathrm{ns}}$ & $-0,71^{* *}$ \\
\hline 12 & 13,9 & 14,2 & $1,27^{*}$ & $0,82^{*}$ & - & $1,04^{\mathrm{ns}}$ & $0,67^{\mathrm{ns}}$ \\
\hline 8 & 13,3 & 13,8 & $0,96^{\mathrm{ns}}$ & $0,17^{* *}$ & - & $0,56^{* *}$ & $-0,27^{* *}$ \\
\hline 17 & 13,1 & 13,4 & $1,27^{\mathrm{ns}}$ & $0,71^{*}$ & - & $0,96^{\mathrm{ns}}$ & $0,84^{\text {ns }}$ \\
\hline 6 & 13,0 & 13,0 & - & - & $1,10^{\mathrm{ns}}$ & $1,01^{\mathrm{ns}}$ & $2,58 * *$ \\
\hline 20 & 12,9 & 12,5 & $0,69^{*}$ & $1,25^{*}$ & & $0,94^{\mathrm{ns}}$ & $2,13 * *$ \\
\hline 4 & 12,9 & 12,9 & - & - & $0,96^{\mathrm{ns}}$ & $0,99^{\mathrm{ns}}$ & $0,61^{\mathrm{ns}}$ \\
\hline 5 & 12,6 & 12,1 & $0,54 * *$ & $1,26^{*}$ & - & $0,94^{\mathrm{ns}}$ & $1,16^{\mathrm{ns}}$ \\
\hline 19 & 12,3 & 12,3 & - & - & $1,21^{* *}$ & $1,17^{*}$ & $1,86^{* *}$ \\
\hline 11 & 12,1 & 12,1 & - & - & $1,23 * *$ & $1,22 * *$ & $1,34^{\mathrm{ns}}$ \\
\hline 14 & 12,1 & 12,1 & - & - & $1,12^{\mathrm{ns}}$ & $1,12^{\mathrm{ns}}$ & $1,06^{\mathrm{ns}}$ \\
\hline 7 & 12,0 & 12,0 & - & - & $0,91^{\mathrm{ns}}$ & $0,87^{\mathrm{ns}}$ & $1,54^{\mathrm{ns}}$ \\
\hline 15 & 12,0 & 11,6 & $0,77^{\mathrm{ns}}$ & $1,29^{*}$ & - & $1,08^{\mathrm{ns}}$ & $0,97^{\text {ns }}$ \\
\hline 2 & 11,7 & 11,7 & - & - & $0,84 * *$ & $0,91^{\mathrm{ns}}$ & $-0,17^{*}$ \\
\hline 3 & 11,2 & 10,8 & $0,77^{\mathrm{ns}}$ & $1,34 * *$ & - & $1,11^{\mathrm{ns}}$ & $0,91^{\text {ns }}$ \\
\hline 18 & 11,1 & 11,1 & - & - & $0,76^{* *}$ & $0,77 * *$ & $0,75^{\text {ns }}$ \\
\hline 16 & 10,9 & 10,9 & - & - & $0,86^{*}$ & $0,86^{*}$ & $0,86^{\text {ns }}$ \\
\hline
\end{tabular}


Quando se considerou o comportamento do tipo bissegmentado, o método de Toler \& Burrows (1998) apresentou valores de $\mathrm{R}^{2}$ maiores que o de Cruz et al. (1989) na maioria dos casos. Já com um único segmento de reta, o de Cruz et al. (1989) mostrou tendência de melhor ajustamento.

Dos 20 genótipos avaliados, dez apresentaram o mesmo tipo de comportamento, enquadrando-se, assim, nos mesmos grupos pelos dois métodos de estabilidade, sendo um no grupo A (20), três no B (9, 19 e 11$)$, três no $\mathrm{C}(4,14$ e 7$)$, dois no $\mathrm{D}(18$ e 16$)$ e um no E (1) (Tabela 3). Entre os quatro primeiros mais produtivos, os de números 9 e 1 enquadraram-se no mesmo grupo (B e E, respectivamente). Segundo Toler \& Burrows (1998), os materiais do grupo B são caracterizados por serem mais responsivos à melhoria da qualidade ambiental, podendo ser indicados para os ambientes com qualidade acima da média geral, e os do grupo $\mathrm{E}$, por mostrarem-se responsivos aos ambientes abaixo da média geral e pouco responsivos aos ambientes de melhor qualidade, podem ser recomendados para os ambientes mais desfavoráveis. Entre os quatro últimos genótipos de menor produtividade, os de números 18 e 16 também se alocaram no mesmo grupo (D). Esses materiais caracterizam-se por serem tolerantes aos ambientes de baixa qualidade, estando, na maioria dos casos, associados a baixas produtividades (Rosse \& Vencovsky, 2000). Por sua vez, os genótipos dos grupos B e E estão sempre associados às maiores produtividades. Quanto aos materiais considerados de padrão de resposta duplamente favorável (grupo A) por Toler \& Burrows (1998), o método linear detectou dois, enquanto o método não-linear detectou quatro.

Comparando os valores de $\mathrm{R}^{2}$ dos genótipos que se situaram nos mesmos grupos pelos dois métodos com aqueles cujo enquadramento se deu em grupos diferentes, o primeiro conjunto mostrou uma média ligeiramente menor em relação ao segundo, 78,5\% e $83,2 \%$, respectivamente (Tabela 3 ). No entanto, essa

Tabela 3. Grupos onde os genótipos foram enquadrados e seus respectivos coeficientes de determinação e as estimativas da qualidade ambiental dos locais de ensaio de caracterização de 1994 da Copersucar, no Estado de São Paulo.

\begin{tabular}{|c|c|c|c|c|c|c|c|}
\hline \multirow[t]{2}{*}{ Genótipo } & \multicolumn{2}{|c|}{ Toler \& Burrows } & \multicolumn{2}{|c|}{ Cruz et al. } & \multicolumn{3}{|c|}{ Qualidade ambiental } \\
\hline & Grupo & $\mathrm{R}^{2}(\%)$ & Grupo & $\mathrm{R}^{2}(\%)$ & Local & $\mu_{j}^{(1)}$ & $\mathrm{Ij}^{(2)}$ \\
\hline 9 & $\mathrm{~B}$ & 79 & $\mathrm{~B}$ & 82 & 1 & $-1,3086$ & $-1,3317$ \\
\hline 13 & $\mathrm{E}$ & 87 & B & 86 & 2 & 2,2720 & 2,3078 \\
\hline 10 & $\mathrm{E}$ & 92 & $\mathrm{C}$ & 88 & 3 & $-0,4322$ & $-0,3027$ \\
\hline 1 & $\mathrm{E}$ & 75 & $\mathrm{E}$ & 69 & 4 & 2,5334 & 2,5168 \\
\hline 12 & E & 83 & $\mathrm{C}$ & 82 & 5 & $-0,6605$ & $-0,6412$ \\
\hline 8 & $\mathrm{E}$ & 54 & $\mathrm{D}$ & 50 & 6 & $-0,4232$ & $-0,5102$ \\
\hline 17 & E & 85 & $\mathrm{C}$ & 82 & 7 & $-1,5444$ & $-1,5591$ \\
\hline 6 & $\mathrm{C}$ & 85 & A & 93 & 8 & $-0,0227$ & $-0,0717$ \\
\hline 20 & A & 78 & A & 82 & 9 & 3,1960 & $-3,2037$ \\
\hline 4 & $\mathrm{C}$ & 75 & $\mathrm{C}$ & 82 & 10 & $-0,1498$ & $-0,1237$ \\
\hline 5 & A & 81 & $\mathrm{C}$ & 84 & 11 & 3,0044 & 2,9328 \\
\hline 19 & B & 88 & B & 89 & 12 & $-0,9694$ & $-1,0127$ \\
\hline 11 & B & 87 & B & 86 & 13 & 0,8970 & 0,9993 \\
\hline 14 & $\mathrm{C}$ & 86 & $\mathrm{C}$ & 85 & & & \\
\hline 7 & $\mathrm{C}$ & 69 & $\mathrm{C}$ & 71 & & & \\
\hline 15 & A & 90 & $\mathrm{C}$ & 89 & & & \\
\hline 2 & D & 80 & E & 89 & & & \\
\hline 3 & A & 88 & $\mathrm{C}$ & 74 & & & \\
\hline 18 & D & 78 & D & 78 & & & \\
\hline 16 & D & 66 & D & 66 & & & \\
\hline
\end{tabular}

${ }^{(1)}$ Efeito ambiental (Toler \& Burrows, 1998). ${ }^{(2)}$ Índice ambiental (Cruz et al., 1989). 
pequena diferença não evidenciou as tendências de comportamento favorecendo um ou outro conjunto quanto ao ajustamento do modelo aos dados. Baseando-se somente no segundo conjunto, no qual o enquadramento foi diferente pelos dois métodos, inferir quais deles forneceram as reais tendências de comportamento dos genótipos é muito difícil, pois, dentro desse conjunto, os valores de $\mathrm{R}^{2}$ foram bastante satisfatórios. Uma alternativa seria basear-se nos respectivos gráficos de regressão. No entanto, como na prática não se conhece todas as variáveis ambientais que possam estar contribuindo mais para essa interação, o emprego da técnica de simulação de dados poderia ser muito útil na escolha do melhor método.

Com relação ao critério de enquadramento pelo método de Cruz et al. (1989), ocorreram algumas situações que merecem ser melhor detalhadas. Por exemplo, os genótipos 13, 11, 18 e 16 apresentaram padrões de resposta para os ambientes favoráveis $\left(\hat{b}_{1}+\hat{b}_{2}\right)$ não-significativos em relação a 1 , indicando que a estimativa de $\hat{b}_{2}$ é estatisticamente igual a zero, de modo que uma única linha de regressão explica satisfatoriamente o comportamento desses materiais diante dos vários ambientes. Já a estimativa de $\hat{b}_{1}$ foi significativa em relação à unidade, sendo nos genótipos 13 e 11 superior a 1, enquadrando-se desse modo no grupo B, e por sua vez, os de números 18 e 16 no grupo $\mathrm{D}$, por ter sido essa estimativa menor que a unidade.

Em relação aos padrões de resposta nos ambientes favoráveis, verifica-se pelo método de Toler \& Burrows (1998) que todos os valores de $\hat{\beta}_{2 \mathrm{i}}$ foram positivos. Porém, no método de Cruz et al. (1989), os valores de $\hat{b}_{1}+\hat{b}_{2}$ dos genótipos 1,8 e 2 foram negativos e significativamente diferentes de 1 (Tabela 2). Em termos práticos, são materiais que respondem negativamente à melhoria das condições ambientais. Há que se ressaltar, também, que os valores de $\mathrm{R}^{2}$ não foram tão elevados (abaixo de $70 \%$ ), exceto quanto ao genótipo 2 , com valor de $89 \%$. Para esses materiais, talvez os modelos empregados não tenham sido os mais adequados.

Outro fato a ressaltar sobre os dois modelos é relacionado à ocorrência de correlação residual entre as estimativas dos parâmetros que discriminam os padrões de resposta nos ambientes favoráveis e desfavoráveis. O desejável é que não ocorra essa associação, sendo essa propriedade vantajosa para o modelo. Em termos práticos, não havendo essa correlação, o melhorista pode escolher um dado genótipo que apresenta uma resposta ideal nos ambientes favoráveis sem ser influenciado pela sua resposta nos ambientes desfavoráveis, sob um mesmo modelo. Rosse (1999) constatou com o modelo de Toler \& Burrows (1998) a existência de associação entre $\hat{\beta}_{1 \mathrm{i}}$ e $\hat{\beta}_{2 \mathrm{i}}$, cuja correlação constitui uma desvantagem para o referido modelo. Pelo método de Cruz et al. (1989), essa associação não mais ocorre. No entanto, a sua ausência culminou com a não-continuidade das linhas de regressão que expressam as respostas dos materiais nos ambientes abaixo e acima da média geral de todos os ambientes. A não-continuidade das linhas de regressão fez com que surgisse um ressalto no ponto $\mathrm{I}_{\mathrm{j}}=0$, o que agronomicamente não é aceito. Desse modo, pode-se visualizar graficamente que em certas situações a produção esperada para um dado ambiente acima da média geral dos ambientes pode ser inferior àquela esperada para um ambiente abaixo desse ambiente médio, tratando-se pois de uma certa incoerência biológica (Duarte et al., 1994). Esse tipo de comportamento foi verificado nos genótipos 6, 20, 11 e 18, que se enquadraram respectivamente nos grupos A, A, B e D.

Uma alternativa para contornar esse tipo de inconveniente metodológico seria fazer com que o ponto de união dos dois segmentos de reta ocorresse num ponto diferente de zero. Biologicamente isso é correto, pois cada genótipo tem um comportamento que lhe é inerente em razão da sua constituição genética, de modo que a sua sensibilidade à mudança ambiental é particular e provavelmente diferente entre os materiais. Portanto, um dado genótipo poderia mudar o seu ângulo de resposta à melhoria do ambiente num ponto à direita ou à esquerda da média ambiental. Esse procedimento foi apresentado por Silva (1998), que constatou valores de $\mathrm{R}^{2}$ maiores em relação aos outros modelos lineares bissegmentados pesquisados.

Com relação ao parâmetro que avalia a qualidade ambiental, para ambos os métodos houve concordância entre os locais que apresentaram valores positivos ou negativos, indicando produtividade ambiental acima e abaixo da média geral, respectivamente (Tabela 3). No entanto, suas magnitudes foram diferentes. 
A razão mais plausível para essa diferença deve-se ao processo de estimação empregado. No modelo linear, embora o índice ambiental $\left(\mathrm{I}_{\mathrm{j}}\right)$ seja estimado separadamente, os próprios dados dos genótipos são utilizados. No modelo não-linear, a qualidade ambiental $\left(\mu_{\mathrm{j}}\right)$ utiliza também os próprios dados dos genótipos, porém o processo de estimação é diferente e mais complexo (processo iterativo de quadrados mínimos não-lineares). Assim, $\mu_{\mathrm{j}}$ é estimado de forma conjunta com os demais parâmetros $\left(\alpha_{i}, \beta_{1 i}\right.$ e $\beta_{2 \mathrm{i}}$ ) utilizando simultaneamente todas as informações contidas na Tabela de dupla entrada, isto é, linhas e colunas, o que caracteriza, segundo Toler \& Burrows (1998), a independência entre as variáveis do modelo de regressão.

No presente trabalho, embora se tenha confrontado dois métodos distintos para estimar parâmetros de estabilidade fenotípica, o enquadramento dos genótipos nos cinco grupos foi de certa forma coerente, isto porque o comportamento que eles apresentaram não dependia do método utilizado e, sim, da sua constituição genética. Além do mais, os valores de $\mathrm{R}^{2}$ foram de uma maneira geral bastante semelhantes. Essa coerência existe em relação aos materiais dos grupos E e D, por exemplo, que caracterizam-se por serem pouco responsivos aos ambientes de melhor qualidade. Por outro lado, os do grupo $\mathrm{C}$ mostram-se responsivos à melhoria da qualidade ambiental, estando mais de acordo com o comportamento dos genótipos dos grupos bissegmentados (A e E).

Embora o modelo de Toler \& Burrows (1998) mostre-se mais adequado, não somente em termos estatísticos mas também em termos gráficos, em relação ao de Cruz et al. (1989), uma decisão para a escolha do melhor modelo a ser utilizado não é tarefa fácil. Um meio de orientar essa decisão seria basear-se nos resultados advindos de um conjunto de dados gerados por simulação.

\section{Conclusões}

1. Não há diferenças marcantes entre os coeficientes de determinação dos genótipos $\left(\mathrm{R}_{\mathrm{i}}^{2}\right)$ pelos dois modelos estudados.
2. As estimativas da qualidade ambiental mostram pequenas diferenças entre suas magnitudes, mas caracterizam igualmente os ambientes acima e abaixo da média geral.

3. Devido às vantagens estatísticas e a maior coerência biológica que o modelo não-linear apresenta sobre o linear, o primeiro deve ser o preferido para inferir sobre a estabilidade fenotípica dos genótipos.

\section{Referências}

CRUZ, C. D.; REGAZZZI, A. J. Modelos biométricos aplicados ao melhoramento genético. Viçosa, MG: UFV, 1994. 390 p.

CRUZ, C. D.; TORRES, R. A. A.; VENCOVSKY, R. An alternative approach to the stability analysis proposed by Silva and Barreto. Revista Brasileira de Genética, Ribeirão Preto, v. 12, n. 3, p. 567-580, 1989.

DUARTE, J. B.; VENCOVSKY, R. Interação genótipos x ambientes: uma introdução à análise "AMMI". Ribeirão Preto: Sociedade Brasileira de Genética, 1999. 60 p. (Monografias, 9).

DUARTE, J. B.; ZIMMERMANN, M. J. O. Comparison of three methods used for the adaptation and phenotypic stability in the common bean (Phaseolus vulgaris L.). Revista Brasileira de Genética, Ribeirão Preto, v. 15, n. 1, p. 125-136, 1992.

DUARTE, J. B.; ROLIM, R. B.; OLIVEIRA, P. M. F.; SOUZA, J. R. Adaptabilidade e estabilidade de rendimento de genótipos de soja [Glicyne max (L.) Merrill], nas condições de Goiás e Distrito Federal. Anais da Escola de Agronomia e Veterinária, Goiânia, v. 24, n. 1, p. 90-104, 1994.

EBERHART, S. A.; RUSSELL, W. A. Stability parameters for comparing varieties. Crop Science, Madison, v. 6, n. 1, p. 36-40, 1966.

GALLANT, A. R. Nonlinear statistical models. New York: J. Wiley, 1987. 610 p.

RAIZER, A. J. Interações genótipos $\mathbf{x}$ ambientes e estabilidade fenotípica em novas variedades de cana-de-açúcar (Saccharum spp.) para o Estado de São Paulo. Piracicaba: Esalq, 1998. 104 p. Dissertação de Mestrado.

RAMALHO, M. A. P.; SANTOS, J. B.; ZIMMERMANN, M. J. O. Genética quantitativa em plantas autógamas: aplicações no melhoramento do feijoeiro. Goiânia: UFG, 1993. 271 p. 
ROSSE, L. N. Modelo de regressão não linear aplicado na avaliação da estabilidade fenotípica em plantas. Piracicaba: Esalq, 1999. 179 p. Tese de Doutorado.

ROSSE, L. N.; VENCOVSKY, R. Modelo de regressão não-linear aplicado ao estudo da estabilidade fenotípica de genótipos de feijão no Estado do Paraná. Bragantia, Campinas, v. 59, n. 1, p. 99-107, 2000.

SILVA, J. G. C. Análise da adaptabilidade através de regressão linear segmentada. Pesquisa Agropecuária Brasileira, Brasília, v. 30, n. 4, p. 435-448, abr. 1995.

SILVA, J. G. C. Análise de adaptabilidade por regressão segmentada com estimação da junção dos segmentos. Pesquisa Agropecuária Brasileira, Brasília, v. 33, n. 7, p. 1013-1029, jul. 1998.

SILVA, J. G. C.; BARRETO, J. N. Aplicação da regressão linear segmentada em estudos da interação genótipo por ambiente. In: SIMPÓSIO DE ESTATÍSTICA APLICA-
DA À EXPERIMENTAÇÃO AGRONÔMICA, 1., 1985, Piracicaba. Anais... Campinas: Fundação Cargill, 1985. p. $49-50$.

STORCK, L. Cultivar stability analysis using a discontinuous bi-segmented model: unbalanced experiments. Pesquisa Agropecuária Brasileira, Brasília, v. 33, n. 5, p. 641-651, maio 1998.

STORCK, L.; VENCOVSKY, R. Stability analysis based on a bi-segmented discontinuous model with measurement errors in the variables. Revista Brasileira de Genética, Ribeirão Preto, v. 17, n. 1, p. 75-81, 1994.

TOLER, J. E.; BURROWS, P. M. Genotypic performance over environmental arrays: a non-linear grouping protocol. Journal of Applied Statistics, Abingdon, v. 25, n. 1, p. 131-143, 1998.

VERMA, M. M.; CHAHAL, G. S.; MURTY, B. R. Limitation of conventional regression analysis: a proposed modification. Theoretical and Applied Genetics, Berlin, v. 53, n. 2, p. 89-91, 1978. 